\title{
High order Adaptive Optics Requirements and feasibility for high contrast imaging
}

\author{
T. Fusco ${ }^{1}$, G. Rousset ${ }^{1}$, J.-L. Beuzit ${ }^{2}$, D. Mouillet $^{3}$, and K. Dohlen ${ }^{4}$ \\ ${ }^{1}$ ONERA, B.P.72, 92322 Chatillon, France \\ ${ }^{2}$ Observatoire de Grenoble, B.P. 53, 38041 Grenoble, France \\ ${ }^{3}$ Observatoire Midi-Pyrénées, B.P. 826, 65008 Tarbes, France \\ ${ }^{4}$ Observatoire de Marseille, B.P. 8, 13376 Marseille, France
}

\begin{abstract}
In the frame of the VLT Planet-Finder project, the phase A system study has demonstrated the feasibility of an extreme adaptive optics system aimed at the direct detection of extrasolar giant planets. The main results of this study are presented in this paper.
\end{abstract}

Keywords. high angular resolution, adaptive optics.

\section{Introduction}

We present here the results of the phase A study of the adaptive optics system for the VLT Planet Finder (VLT-PF) instrument (Beuzit et al. 2005). The main scientific objective of VLT-PF is the direct detection of photons coming from giant extrasolar planets (between 1 and 20 Jupiter masses). Any detection will then be followed by a first characterisation of the planet atmosphere (clouds, dust content, methane, water absorption...). In addition, the survey of an extended number of stars (typically a few hundreds) is mandatory in order to perform meaningful statistical studies. Such extremely challenging scientific objectives directly translate into a relatively complex high constrast instrument. Coronagraphic and smart imaging (differential imaging for instance) capabilities are essential to reach the high constrat (close to the optical axis) required for direct extrasolar planet detection. From the ground, the core of any high contrast instrument is an extreme adaptive optics (XAO) system correcting for the perturbation induced by the atmospheric turbulence as well as for the internal aberrations of the instrument itself.

Based on the specifications provided by the astronomers (Moutou et al. 2005) and on the constraints of the high contrast instrumentation, the XAO must fulfill the following three high level requirements: 1) ensure the measurement and correction of the turbulent phase perturbations, of the telescope and system common optics aberrations, of the noncommon path aberrations (main AO loop); 2) ensure an extremely high stability (at low temporal frequency) of the optical axis at the level of the coronagraphic mask (Auxiliary Sensor $[\mathrm{AS}]$ ); 3) ensure the measurement and the correction of any pupil motion (Pupil Motion Sensor [PMS]).In the following sections, we will mainly focus on the main AO loop design. The two other requirements (essential to ensure optimal coronagraphic extinction and, in fine, planet detection performance) will only be briefely addressed.

\section{AO loop performance in the focal plane}

The VLT-PF system aims at the detection of extremely faint sources (giant extrasolar planets) in the vinicity of bright stars. Such a challenging goal requires the use of a coronagraphic device to cancel out the flux coming from the star itself. Even if additionnal 
smart imaging techniques are added to reach the required contrast for extrasolar planet detection (typically $10^{-6}-10^{-7}$ in contrast), the coronagraphic image is a basis for the AO optimisation. Moreover, unlike classical AO systems, residual variance or Strehl ratio are not sufficient anymore to optimize the system and to derive the pertinent trade-offs. They have to be replaced by a more accurate parameter which can provide information on the coronagraphic image shape in the focal plane. The purpose of the coronagraph is to remove the coherent light coming from the on-axis guide star (GS). Therefore one can analytically define a "perfect coronagraph" using the following equations:

$$
I_{\text {corona }}(\rho)=\left\langle\left|\mathrm{FT}\left[P(r) A(r) e^{i \varphi_{\text {res }}(r)}-\sqrt{\exp \left[-\sigma_{\varphi+\log (A)}^{2}\right]} P(r)\right]\right|^{2}\right\rangle
$$

where $\langle$.$\rangle stands for a statistical average and with A(r)$ the wavefront amplitude, $\varphi_{\text {res }}(r)$ the residual phase after AO correction, $P(r)$ the pupil function and $\sigma_{\varphi+\log (A)}^{2}$ the residual variance gathering phase and log-amplitude effects. When only a partial correction is performed, the coherent peak is removed and only the uncoherent light (the residual uncorrected speckles) remains. In that case, it is easy to show that, as a first approximation (first order expansion) the coronagraphic image is proportional to the residual phase power spectral density.

\section{Balance of an error budget}

The whole AO study is based on the balance of an error budget. A first approximation (first order expansion) of this coronagraphic shape is given by the residual phase power spectral density (PSD) as shown in Section 2. Therefore, the global error budget for the AO system can be summarized as follow:

$$
\begin{aligned}
\mathbf{P S D}_{\text {res }}= & \underbrace{\mathbf{P S D}_{\text {scint }}+\mathbf{P S D}_{\text {diff }}+\mathbf{P S D}_{\text {refrac }}+\mathbf{P S D}_{\text {aniso }}}_{\text {atmospheric limitation }} \\
& +\underbrace{\mathbf{P S D}_{\text {fit }}+\underbrace{\mathbf{P S D}_{\text {tem } p}+\mathbf{P S D}_{\text {alias }}+\mathbf{P S D}_{\text {noise }}}_{\text {low order residual error }}}_{\text {AO loop residual error }}+\underbrace{\mathbf{P S D}_{\text {calib }}+\mathbf{P S D}_{\text {aberr }}}_{\text {calibration errors }}
\end{aligned}
$$

A complete description can be found in Fusco et al. 2005b. $\mathbf{P S D}_{\text {res }}$ is expressed in terms of residual variance per spatial frequency in order to highlight the range of spatial frequencies which is affected by each error item. The otimisation of this error budget will be performed, keeping in mind three main criteria:

- The corrected area, i.e. the focal plane area where the image contrast is significantly improved by the AO system. It mainly drives the choice of the number of actuators (the correction area is equal to $\lambda_{i m} / d$ in diameter, where $d$ is the actuator spacing). Considering the typical targets which will be observed by VLT-PF and the imaging wavelengths (J, H and $\mathrm{K}$ bands), this area has to be larger than 0.8 arcsec in diameter.

- The detectivity level, i.e. the capability of the whole system to detect the planet signal. This level is affected by the AO loop errors (temporal, noise, aliasing...) which evolve rapidly with time and can be calibrated using differential imaging and a reference PSF. It can also be degraded by the telescope and the system high spatial frequencies and non-common path aberrations which slowly evolve with time and represent the ultimate limitation for the differential imaging and reference PSF subtraction techniques. The minimisation of the slowly variable defects implies the measurement and the correction 
of non-common path aberrations (see Section 6) as well as the stabilisation of the optical beam during a whole observation sequence (see Section 7.2).

- The system sensitivity. This criterion is driven by the number of stars to be observed but highly depends on the detectivity level and the corrected area size. Indeed, the larger the corrected area the smaller the available flux per individual measurement zones (subaperture in the case of a SH device for instance). In addition, increasing the detectivity level implies a reduction in terms of temporal and noise errors, which leads to a faster system working on brighter guide stars for wavefront sensing. A first trade-off between scientific goals and system requirements has led to a limiting magnitude of 8 in $\mathrm{H}$-band (corresponding to magnitudes 10-11 for visible band, depending on the GS types) for the system, implying that the detectivity capabilities have to remain optimum up to this magnitude.

In addition to this three scientific criteria, other constraints have to be taken into account during the instrument design: 1) use of well-proven technologies if possible; 2) new developments for critical issues only, with associated experimental validations; 3 ) the system has to be built on a tight schedule ( 5 years typically) with finite manpower and budget. These last three points are essential to minimize the risk factor during the instrument realisation.

\section{Atmospheric limitations}

The atmospheric limitations (Roddier \& Roddier 1986) which gather all the errors due to propagation effects (scintillation, diffraction, anisoplanatism and differential refraction effects). Full correction of scintillation effects would only lead to an equivalent reduction of the phase variance smaller than $20 \mathrm{~nm}$ rms. In comparison of the small gain in performance, the system complexity is highly increased (two DM, measurement device, reconstruction process...). Considering the small expected gain, even if the scintillation is fully corrected (which is far from being obvious) it has been decided not to consider a scintillation corrector for the VLT Planet Finder instrument.

\section{AO loop residual errors}

The AO loop residual errors which gather all the "classical" AO loop errors and can be sub-divided in

- The fitting errors (high order modes not corrected by AO, i.e. frequencies higher than the AO spatial cut-off frequency). Considering the Planet Finder Top Level Specifications, the minimisation of the global error budget is not the only pertinent criterion, the spatial repartition of the errors has also to be taken into account. In particular, the detectivity performance is also linked to the capacity of the AO system to "clean up the PSF" (i.e. to be as close as possible to the diffraction pattern) in an area of more than 0.75 " in radius from the optical axis. This corrected area (Corr) in the focal plane is directly linked to the inter-actuator distance $\left(d=D /\left(n_{a c t}-1\right)\right.$, where nact is the linear number of actuators in the telescope diameter $D$ ) with the following relation: corr $=\lambda_{i m} / 2 d$. Decreasing $d$, that is increasing the number of actuators, has however consequences in term of the system limiting magnitude. Indeed, the larger the number of DM actuators, the larger the number of WFS sub-apertures and hence the smaller the number of available photons per sub-aperture and per frame.

- Low order modes (corrected by AO) gathering the aliasing, temporal and noise errors. 
i Aliasing error: these effects are due to high spatial frequencies seen as low ones by the WFS device. The aliasing error is directly linked to the fitting error and in the specific case of SH WFS it corresponds to roughly $40 \%$ of the total fitting error variance. It dramatically increases the PSF residuals in its corrected area. A concept has been recently proposed (Poyneer \& Macintosh 2004) to significantly reduce the aliasing effect. This device has been deeply studied and optimised with respect to the system and turbulence characteristics (spectral bandwidth, WFS sampling, turbulence ...). In addition, an experimental validation of the concept has been conducted using the ONERA AO bench (see Fusco et al. 2005a), in closed loop and with turbulence. A gain brought by a filtering device has been clearly demonstrated and it has been shown that the experimental results are in good agreement with the simulation, which validates the potentiality of the concept and its use in the VLT-PF design.

ii Temporal error: the AO loop temporal behaviour depends on each AO components (detector integration time, readout noise, real-time computations of commands from WFS data, numerical corrector, digital-analog converter, high voltage amplifier, DM actuator temporal response) and turbulence characteristics. The optimal way to deal with all these parameters is to design a Kalman filter based control algorithm (Petit set al. 2004). Nevertheless, practical implementation of such a control law is complex and requires more computing power, especially for high order systems. This leads to a significant increase of the RTC complexity. An hybrid solution has been considered to deal with this problem. An optimal modal gain integrator has been chosen to control high order modes while a Kalman filter has to be considered for tip-tilt modes to optimally correct turbulence and vibration effects

iii WFS measurement error: a comparison between SH and Pyramid WFS in the frame of the VLT-PF AO system has been performed. In both cases, performance, required calibrations and optimizations, as well as fundamental limitations have been identified and quantified. From the WFS performance, stability, complexity and risk evaluation, a spatially filtered SH WFS, combined with a new optimized slope estimation algorithm (Nicolle et al. 2004), has been chosen as a baseline for the VLT-PF AO system. It allows us to reach a limiting magnitude of around 10-11 (depending on GS type and CCD performance (Fusco et al. 2004))

\section{Calibration error}

The calibration errors (Fusco et al. 2003) that gather the AO loop mis-calibration (interaction matrix and reference slopes) and the mis-calibration of the non-common path aberrations (NCPA). Effects of mis-aligment on system performance have been studied leading to tight specifications on system stability (pupil conjugaison between DM and WFS, pupil motion ...). NCPA will be measured using a phase diversity approach Sauvage et al. 2005 and corrected in closed loop through a modification of the WFS references.

\section{Auxiliary devices}

\subsection{IR Tip-Tilt sensor}

The average image position (in other words the optical axis position) on the coronagraphic mask is a main specification for the VLT-PF system. The required accuracy for the mean image position is 0.5 mas or better. The global error for the average image position mainly depends on the differential refraction effect (between VIS and IR wavelengths) and the 
differential thermal or mechanical effects (between WFS and imaging paths). Considering the requirement, an open loop model of each differential evolution will not be accurate enough (considering all the possible parameters involved) to reach the absolute position performance. Therefore, to ensure that the specification will be fullfilled, an auxiliary IR tip-tilt sensor (AS) at the level of the coronagraphic mask has been proposed. This sensor will be coupled with a differential tip-tilt mirror (DTTM) located in an pupil plane in the WFS arms.

\subsection{Pupil motion sensor}

Pupil stability is a major issue to ensure the VLT-PF performance. The pupil has to remain motionless during the whole observation process. When located after the Nasmyth focus of the telescope, this stability requirement implies a pupil de-rotator and a pupil re-centering device. It has been shown that a pupil shift of $1 \%$ of the pupil diameter or a pupil rotation of 1 degree will reduce by a factor of 1.5 to 2 (for typical conditions at the VLT) the detection capability of a coronagraphic + differential imaging system. This

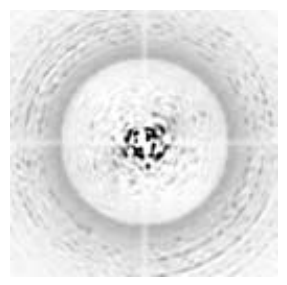

differential image

$$
I\left(\lambda_{1}\right)-I\left(\lambda_{2}\right)
$$
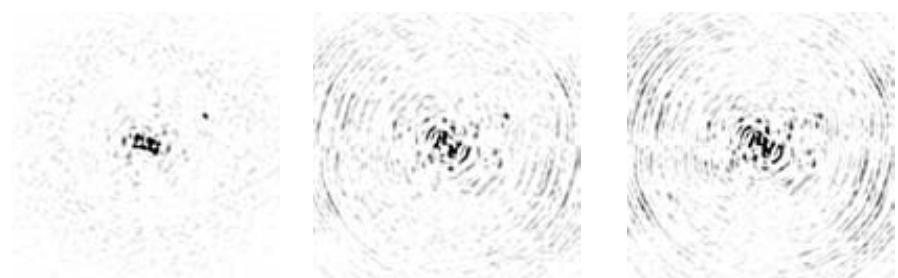

motionless pupil pupil motion $=0.6 \%$ pupil motion $=1.2 \%$ Differential image + calibration on reference star

$$
\left[I\left(\lambda_{1}\right)-I\left(\lambda_{2}\right)\right]-\left[\operatorname{Re} f\left(\lambda_{1}\right)-\operatorname{Ref}\left(\lambda_{2}\right)\right]
$$

Figure 1. [Left] Differential coronagraphic (4-quadrant) image $\left(\lambda_{1}=1.56 \mu \mathrm{m}, \lambda_{2}=1.59 \mu \mathrm{m}\right)$, [Right] differential coronagraphic image + reference subtraction: pupil shift between object and reference star $=0,0.6$ and $1.2 \%$ of the full pupil. The companion $\left(\Delta_{m}=15\right.$, separation $=0.6$ arcsec) is really distinguishable from residual fixed speckles for a fixed pupil.

had led to impose a pupil stability in translation better than 0.2 (goal $0.1 \%$ ) of the full VLT pupil. This performance is achieved using a pupil tip-tilt mirror located close to the entrance focal plane of the VLT-PF. This mirror is controlled by a pupil motion sensor (PMS). The PMS directly uses the SH-WFS data to measure pupil motion. Because pupil motion is rather slow, a measurement-correction process has to be performed typically every minute which ensures a good SNR on the PMS data.

\section{Global system design}

A global trade-off from all the points mentioned above (combined with optical design, technological aspect, cost and risk issues) leads to the following AO system main characteristics:

- A 41x41 actuator DM of $180 \mathrm{~mm}$ diameter, located in a pupil plane with an interactuator stroke $> \pm 1 \mu m$ (mechanical), a maximum stroke $> \pm 3.5 \mu m$ (mechanical), plus a 2-axis TTM with a \pm 0.5 mas resolution.

- 40x40 Shack-Hartmann WFS, with a spectral range between 0.45 and $0.95 \mu m, 6 x 6$ pixels per sub-aperture (Shannon sampling @ $0.65 \mu \mathrm{m}$ ), a focal plane filtering device with variable size (from $\lambda / d$ to $3 \lambda / d$ at $0.7 \mu m$ ) and a temporal sampling frequency $1 \mathrm{kHz}$ (goal $1.5 \mathrm{kHz}$ ). The foreseen detector is a $256 \times 256$ pixels Electron Multiplication CCD detector with a read-out-noise $<1 e^{-}$and a 1.4 excess photon noise Fusco et al. 2004. 
- Mixed numerical control law with a Kalman filter law for Tip-Tilt control and an Optimal Modal Gain Integrator law for DM control. The global AO loop delay has to be lower than $1 \mathrm{~ms}$ (goal $666 \mu \mathrm{s}$ ).

- Non-common path aberrations: off-line measurements and on-line compensation using a phase diversity algorithm.

- Auxiliary IR tip-tilt sensor and pupil sensor to measure and xcorrect for optical axis and pupil displacement.

End-to-end simulations have been performed with this conceptual design. Results are summarized in Table 1. Using these AO simulations in a complete systeme model (in-

\begin{tabular}{|c|c|c|c|c|c|c|c|c|c|c|}
\hline Seeing conditions & & $0.65 "$ & & $\|$ & & 0.85 & & $\|$ & \multicolumn{2}{|c|}{$1.05 "$} \\
\hline atial & ow f & eq. $\mid$ & $\operatorname{sh} f$ & & 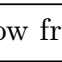 & .11 & & & $f$ & \\
\hline Mag 9 & 46 & 1 & 51 & $\|$ & 54 & 1 & 67 & $\|$ & 67 & 81 \\
\hline Mag 11 & 64 & 1 & 51 & $\|$ & 73 & 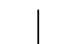 & 67 & $\|$ & 90 & 81 \\
\hline
\end{tabular}

Table 1. AO error budget (in $\mathrm{nm} \mathrm{rms}$ ) for various seeing and GS flux conditions. In each case, both low spatial frequency error (i.e corrected by AO) and hig spatial frequencies error (fitting error) are given.

cluding AO, coronagraphy, differential imaging and detectivity process) it has been shown that these values satisfy the VLT-PF high level requirements alowing direct detection of hot Jovian-like planets. In conclusion, an AO for Planet Finder represent a large step forward both in terms of system components and calibration procedures, nevertheless a complete analysis (with a detailed error budget) has shown that a AO system fulfilling all the requirement mandatory for the direct detection of hot Jupiter like planet is feasible in a reasonable time scale (5 years) with tried and tested technologies.

\section{References}

J.-L. Beuzit et al. "A planet Finder instrument for the VLT", This conf., 2005

C. Moutou et al. "Science case for the next generation VLT AO instrument Planet Finder", This conf., 2005

T. Fusco et al. "Conceptual design of an extreme AO dedicated to extra-solar planet detection by the VLT-planet Finder intrument", 5903, Proc. Soc. Photo-Opt. Instrum. Eng., 2005

F. Roddier and C. Roddier, "Noao infrared adaptive optics program ii: modeling atmospheric effects in adaptive optics systems for astronomical telescopes," in Advanced Technology Optical Telescopes III, 628, Proc. Soc. Photo-Opt. Instrum. Eng. (Washington), 1986

C. Petit et al. "Kalman filter based control loop for adaptive optics," in Advancements in Adaptive Optics, 5490, SPIE, 2004

L. A. Poyneer and B. Macintosh, "Spatially filtered wave-front sensor for high-order adaptive optics," J. Opt. Soc. Am. A 21(5), pp. 810-819, 2004

T. Fusco, M. Nicolle, G. Rousset, V. Michau, J.-L. Beuzit, and D. Mouillet, "Optimisation of Shack-Hartmann-based wavefront sensor for XAO system," in Advancements in Adaptive Optics, 5490, Proc. Soc. Photo-Opt. Instrum. Eng., 2004

T. Fusco et al., "Closed-loop experimental validation of the filtered Shack-Hartmann," Opt. Lett. 29, pp. 1255-1257, Jun. 2005

M. Nicolle et al. "Improvement of shack-hartmann wavefront sensor measurement for extreme adaptive optics," Opt. Lett. 29, pp. 2743-2745, Dec. 2004

T. Fusco, G. Rousset, and A. Blanc, "Calibration of AO system. Application to NAOSCONICA," in Science with Adaptive Optics, W. Brandner and M. Kasper, eds., SpringerVerlag, 2004. Sept. 2003, Garching, Germany

J.-F. Sauvage et al. "Fine calibration and pre-compensation of non-common path aberrations for high performance AO system," in This conference, 2005 